\title{
High-mobility group nucleosome-binding domain 2 protein inhibits the invasion of Klebsiella pneumoniae into mouse lungs in vivo
}

\author{
SHUANG ZHENG* , LAIBIN REN* , HENG LI, XIAOFEI SHEN, XIAOLONG YANG, NA LI, \\ XINYUAN WANG, XIAOJUAN GUO, XIAOYING WANG and NING HUANG \\ Department of Pathophysiology, West China School of Preclinical Sciences and Forensic Medicine, \\ Sichuan University, Chengdu, Sichuan 610041, P.R. China
}

Received June 23, 2014; Accepted January 29, 2015

DOI: $10.3892 / \mathrm{mmr} .2015 .3464$

\begin{abstract}
Since bacterial invasion into host cells is a critical step in the infection process and the predominance of multiple-antibiotic-resistant Klebsiella (K.) pneumoniae strains, using molecular agents to interfere with $K$. pneumoniae invasion is an attractive approach for the prevention of infection and suppress the immune inflammatory response. In previous studies by our group, high-mobility group nucleosome-binding domain 2 (HMGN2) protein was shown to exhibit anti-bacterial activity in vitro. The objective of the present study was to investigate the effects of HMGN2 protein on the invasion of $K$. pneumoniae 03183 in vivo. The results showed that pre-treatment with $128 \mu \mathrm{g} / \mathrm{ml}$ HMGN2 significantly reduced $K$. pneumoniae 03183 invasion into mouse lungs and increased the mRNA expression of CXCL1 and LCN2 within $2 \mathrm{~h}$. Immunohistochemical staining showed that F-actin expression was significantly decreased, and fluorescence microscopy and western blot analysis further demonstrated that HMGN2 significantly blocked K. pneumoniae 03183 -induced actin polymerization. These changes implied that HMGN2 may provide protection against $K$. pneumoniae 03183 infection in vivo.
\end{abstract}

Correspondence to: Professor Xiaoying Wang or Professor Ning Huang, Department of Pathophysiology, West China School of Preclinical Sciences and Forensic Medicine, Sichuan University, No.17, Third Section of Renmin South Road, Chengdu, Sichuan 610041, P.R. China

E-mail: wangxiaoyingzs@126.com

E-mail: huangningzs@126.com

*Contributed equally

Key words: High mobility group nucleosome-binding domain 2, Klebsiella pneumoniae, invasion, F-actin, cytokines

\section{Introduction}

Klebsiella pneumoniae is a species of aerobic gram-negative bacteria, which primarily inhabits the intestines, respiratory system and urinary system of humans and animals (1). It is well known as a cause of community-acquired pneumonia (2). Resistance of $K$. pneumoniae to antibiotics is an increasingly severe problem resulting in an urgency for the development of novel types of antibiotic (3).

Invasion of host cells is the singular most important mechanism that bacterial pathogens employ to avoid rapid clearance by the host immune system (4). A common and recurring target of pathogens is the cytoskeleton of host cells, which is utilized by these microbes for purposes that include attachment, entry into cells, movement within and between cells, vacuole formation and remodelling, and avoidance of phagocytosis (5). Actin has two forms, one is a monomer (G-actin) and the other a multimer (F-actin). Normally, the progress of polymerization into F-actin (6) and depolymerization into G-actin is balanced to regulate actin activity, as only polymeric actin has biological effects $(7,8)$. Diverse bacteria invade non-phagocytic cells by stimulating the endogenous uptake processes, including phagocytosis and macropinocytosis. Actin polymerization is central to these two processes, driving plasma membrane extensions that engulf external cargo (9).

Cytokines and chemokines are critical molecules in response to pathogen invasion and are involved in almost every facet of immunity and inflammation (10,11). Interleukin (IL)-6 has been identified to enhance survival of pneumonia sepsis caused by K. pneumoniae via increasing neutrophil death (12). CXCL1 has been demonstrated to be able to enhance resistance to $K$. pneumonia in mice $(13,14)$. LCN2 may inhibit bacterial growth by sequestering the iron-binding bacterial siderophores and blocking bacterial access to iron $(15,16)$.

Anti-microbial peptides (AMPs) are active at physiological concentrations under conditions prevailing in the tissues of origin (17). High-mobility group nucleosome-binding domain 2 (HMGN2) protein, a member of the HMGN family, binds specifically to nucleosomes (18) in the cell nucleus of vertebrate and invertebrate organisms and has a role in global DNA repair (19). Previous studies by our group revealed that 
HMGN2 protein had anti-bacterial activity and is therefore considered as a potent AMP $(20,21)$. Is was able to inhibit the gene expression and replication of hepatic B virus in vitro (22) and also exhibited anti-cancer potency in HeLa cells (23). Further studies by our group demonstrated that pre-treatment of $K$. pneumoniae 03183 with $128 \mu \mathrm{g} / \mathrm{ml}$ HMGN2 significantly inhibited the internalization of $K$. pneumoniae 03183 into human bladder carcinoma T24 cells by decreasing K. pneumoniae-induced actin polymerization $(24,25)$.

HMGB1, a member of the HMG superfamily, stimulates pro-inflammatory cytokine synthesis in human monocytes (26) and contributes to the occurrence of rheumatoid arthritis, systemic lupus erythematosus and polymyositis $(27,28)$. HMGN1, like HMGN2, a member of the HMGN subfamily, also induces pro-inflammatory cytokine production in vitro and in vivo (29). In the present study, K. pneumoniae 03183 bacteria were pre-treated with $128 \mu \mathrm{g} / \mathrm{ml}$ HMGN2 and the effects of HMGN2 on K. pneumoniae 03183 -induced actin polymerization as well as on the expression of the K. pneumoniae 03183-associated cytokines lipocalin-2 (LCN2), chemokine (C-X-C motif) ligand 1 (CXCL1) and IL-6 in mouse lungs were assessed.

\section{Materials and methods}

Chemicals. Recombinant human HMGN2 was prepared as previously described (24). $1 \%$ bovine serum albumin (BSA) was obtained from Thermo Fisher Scientific (Waltham, MA, USA).

Bacteria and culture conditions. K. pneumoniae strain 03183 was isolated from a sample from a patient with a urinary tract infection at the Department of Medicine, West China Hospital affiliated to Sichuan University (Chengdu, China). K. pneumoniae 03183 was grown in Luria-Bertani (LB) broth (Oxoid Ltd., Basingstoke, UK) for $14-16 \mathrm{~h}$ at $37^{\circ} \mathrm{C}$ until reaching the middle of the logarithmic growth phase. The concentration of bacteria in the LB broth was determined by measuring the absorbance at $650 \mathrm{~nm}$. Later, K. pneumoniae 03183 was pre-treated with $128 \mu \mathrm{g} / \mathrm{ml} \mathrm{HMGN} 2$ and $1 \%$ BSA separately for $2 \mathrm{~h}$ at $37^{\circ} \mathrm{C}$. The inoculum was pelleted by centrifugation at 7,000 $\mathrm{x} \mathrm{g}$ for $2 \mathrm{~min}$ and resuspended in phosphate-buffered saline (PBS; Chengdu Kelong Chemical Co., Ltd., Chengdu, China) to obtain the desired concentration $\left(10^{9}\right.$ colony-forming units).

Animals and treatment. A total of 24 female C57BL/6 mice (6-8 weeks old with similar weights) were obtained from the Experimental Animal Center of Sichuan University (Chengdu, Sichuan). The mice were fed a normal diet and were kept at room temperature with a cycle of $12 \mathrm{~h}$ light and $12 \mathrm{~h}$ dark. They were randomly divided into four groups: In the PBS group, mice were transtracheally inoculated with PBS; in the HMGN2 group, mice were transtracheally inoculated with K. pneumoniae 03183 pre-treated with $128 \mu \mathrm{g} / \mathrm{ml}$ HMGN2; in the BSA group, mice were transtracheally inoculated with $K$. pneumoniae 03183 pre-treated with $1 \%$ BSA; in the $K$. pneumoniae group, mice were transtracheally inoculated with untreated K. pneumoniae 03183. Each experimental group contained six animals. The study was approved by the ethics committee of West China College of Preclinical Sciences and Forensic Medicine, Sichuan University.

In vivo invasion assay. Each animal was anesthetized with by i.p. injection of $10 \%$ chloral hydrate (Chengdu Kelong Chemical Co., Ltd.). The trachea was exposed, and the respective inoculum was administered via a sterile 26-gauge needle. The skin incision was closed by surgical suture. After $2 \mathrm{~h}$, the lung was aseptically removed, $0.1 \mathrm{mg}$ of gentamicin was applied for $30 \mathrm{~min}$ to kill all extracellular bacteria, the lung was washed briefly using $100 \mu 1$ PBS and then homogenized in PBS according to a previously published procedure (4). Homogenate dilutions were plated at $\sim 1 \times 10^{5}$ cells $/ \mathrm{ml}$ on LB plates and incubated overnight at $37^{\circ} \mathrm{C}$ for colony counts.

Histopathological examination and immunofluorescence of lung sections. Each animal was anesthetized and inoculated transtracheally with inoculum or PBS. After $2 \mathrm{~h}$, the lung was aseptically removed, immersed in $10 \%$ formalin fixative (Chengdu Kelong Chemical Co., Ltd.) and processed for histological examination. The lung tissue was embedded in paraffin wax (Chengdu Kelong Chemical Co., Ltd.) and cut into 4-6 mm-thick sections using a microtome. After this, the sections were stained with hematoxylin and eosin (Beyotime Institute of Biotechnology, Nanjing, China) by standard techniques (30). To observe the expression of F-actin by immunohistochemistry, sections of uninfected and infected lungs were prepared using standard methods (31). Each section was immerged in $30 \% \mathrm{H}_{2} \mathrm{O}_{2}$ (Chengdu Kelong Chemical Co., Ltd.)/distilled water (1:10) for $10 \mathrm{~min}$ at room temperature. Each section was blocked in PBS containing 5\% BSA for $10 \mathrm{~min}$ at room temperature following microwave antigen retrieval, which involved heating the sections in citrate buffer at $92-98^{\circ} \mathrm{C}$ for $10-15 \mathrm{~min}$. The sections were then incubated with monoclonal anti-mouse F-actin (1:100; ab130935; Abcam, Cambridge, UK) diluted 1:100 in PBS with 5\% BSA overnight at $4^{\circ} \mathrm{C}$. Sections were incubated for $30 \mathrm{~min}$ at $37^{\circ} \mathrm{C}$ with biotinylated mouse anti-mouse immunoglobulin $\mathrm{IgG}$ (1:100, ZB-2055; ZSGB-BIO, Beijing, China), then streptavidin-horseradish peroxidase (HRP) (1:100; ZB2404; HongYue ChuangXin Technology Co., Ltd., Bejing, China) was applied for $30 \mathrm{~min}$ at $37^{\circ} \mathrm{C}$, using a diaminobenzidine kit (ZSGB-BIO) to display the results. For immunostaining, sections were fixed and blocked in the same manner as stated above and incubated with monoclonal mouse F-actin antibody diluted 1:50 in PBS with $5 \%$ BSA overnight at $4^{\circ} \mathrm{C}$. Sections were then incubated with monoclonal biotinylated $\mathrm{Cy} 3$ goat anti-mouse $\operatorname{IgG}$ (1:50; C5585; Sigma-Aldrich) for $60 \mathrm{~min}$ at $37^{\circ} \mathrm{C}$ and DAPI (for nuclear staining; Sigma-Aldrich, St Louis, MO, USA) for $30 \mathrm{~min}$ at room temperature in the dark, mounted and viewed using an Axio Scope A1 microscope (Zeiss, Oberkochen, Germany).

Quantification of F-actin by western blot analysis. To examine F-actin protein by western blot analysis, lung tissues were homogenized with lysis buffer $(10 \mathrm{mM}$ Tris, $\mathrm{pH} 8.0$; $1 \mathrm{mM}$ EDTA; $400 \mathrm{mM} \mathrm{NaCl}$; $10 \%$ glycerol; $0.5 \% \mathrm{NP}-40$; $5 \mathrm{mM}$ sodium fluoride; $0.1 \mathrm{mM}$ phenylmethylsulfonylfluoride; and $1 \mathrm{mM}$ dithiothreitol) $(32,33)$. The lysates were centrifuged $\left(12,000 \mathrm{x} \mathrm{g}, 20 \mathrm{~min}, 4^{\circ} \mathrm{C}\right)$ and the supernatants 


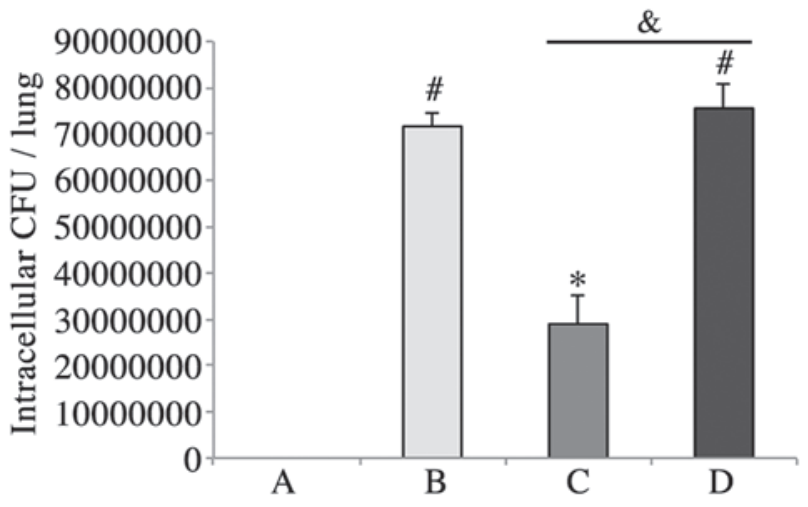

Figure 1. Effect of HMGN2 on $K$. pneumoniae 03183 invasion (A) Phosphate-buffered saline-treated group; (B) group inoculated with bovine serum albumin-treated $K$. pneumoniae 03183; (C) group inoculated with HMGN2-treated $K$. pneumoniae 03183; (D) group inoculated with untreated $K$. pneumoniae 03183 . ${ }^{*} \mathrm{P}<0.05,{ }^{\#} \mathrm{P}<0.01$, compared with $\mathrm{A} ;{ }^{\&} \mathrm{P}<0.05$ error bars represent the mean + standard deviation. HMGN2, high-mobility group nucleosome-binding domain 2; CFU, colony-forming units.
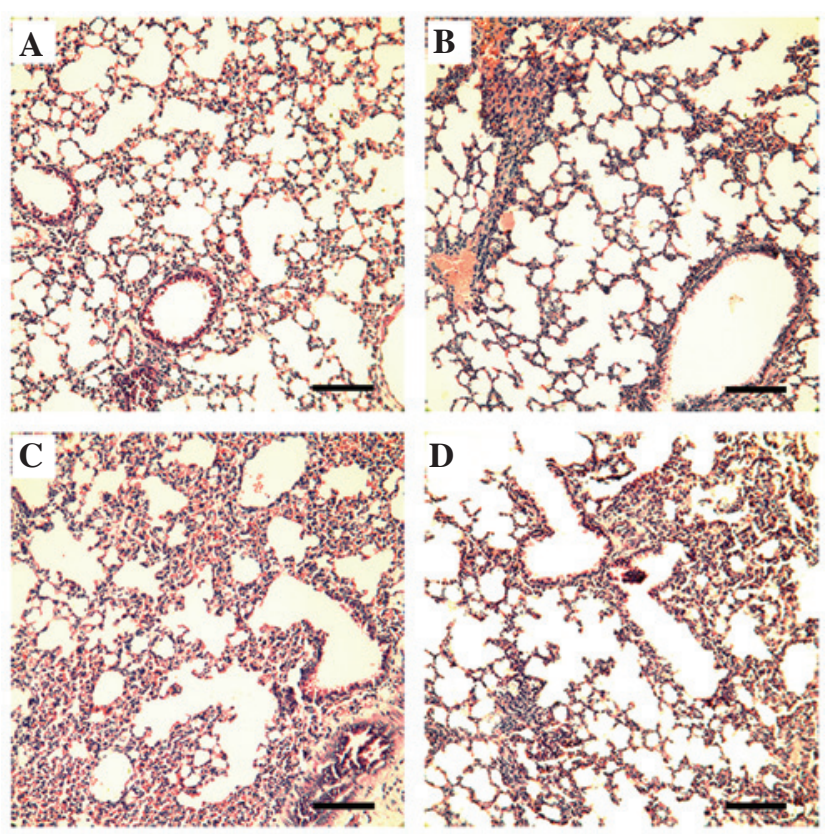

Figure 2. Hematoxylin and eosin staining of lung tissue (magnification, x100 scale bar, $40 \mu \mathrm{m}$ ). (A) Phosphate-buffered saline-treated group; (B) group inoculated with bovine serum albumin-treated $K$. pneumoniae 03183 (C) group inoculated with high-mobility group nucleosome-binding domain 2-treated K. pneumoniae 03183; (D) group inoculated with untreated K. pneumoniae 03183

were collected. Protein concentrations were determined using a BCA-1 bicinchoninic acid protein assay kit (Sigma-Aldrich), and equal amounts of protein $(50 \mu \mathrm{g})$ were separated on precast $10 \%$ SDS-polyacrylamide gels (Beyotime Institute of Biotechnology), electrotransferred to polyvinylidene fluoride membranes (Merck Millipore, Darmstadt, Germany) and blocked with 5\% skimmed milk (Sigma-Aldrich) at room temperature for $1 \mathrm{~h}$. The membranes were incubated with primary antibody for F-actin (1:500) or GAPDH (1:500; AG019; Beyotime Institute of Biotechnology, Haimen, China) at $4^{\circ} \mathrm{C}$ overnight. Following repeated washing, the membranes were incubated with HRP-conjugated anti-mouse
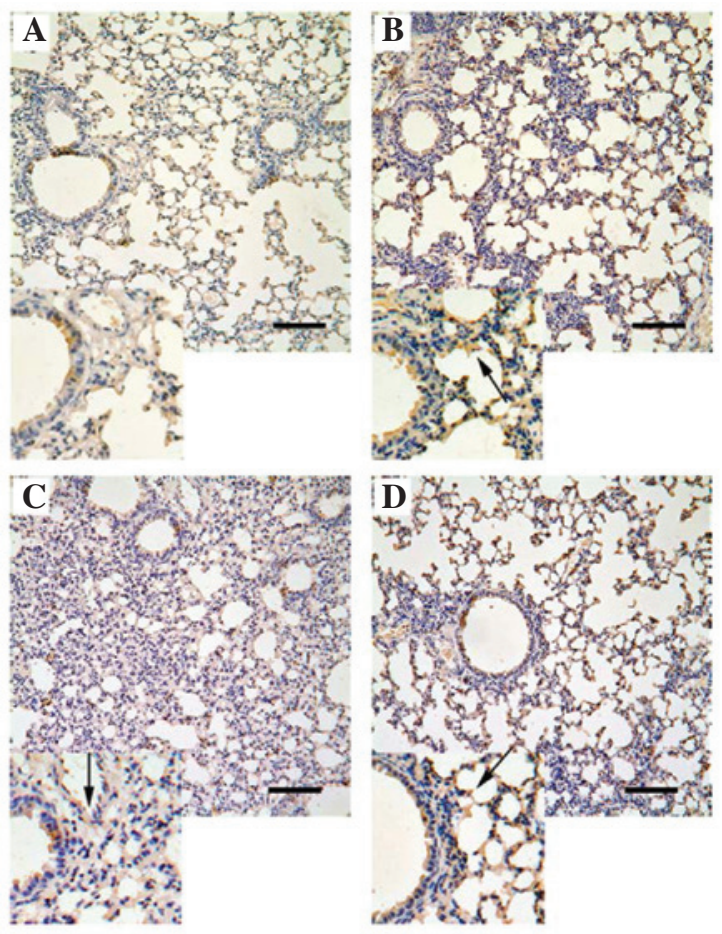

$\mathbf{E}$

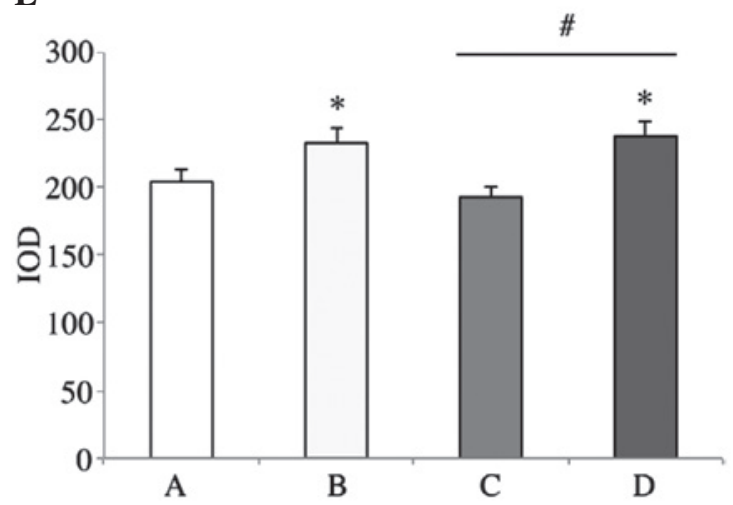

Figure 3. Immunohistochemistry of lung tissue (magnification, x100; insets, $\mathrm{x} 400$; scale bar, $40 \mu \mathrm{m}$; black arrows indicate positive F-actin expression). (A) Phosphate-buffered saline-treated group; (B) group inoculated with bovine serum albumin-treated K. pneumoniae 03183; (C) group inoculated with high-mobility group nucleosome-binding domain 2-treated $K$. pneumoniae 03183; (D) group inoculated with untreated K. pneumoniae 03183; (E) Quantification of A-D.Bars represent the means of IOD of F-actin staining. Black arrows indicate positive F-actin expression. " $\mathrm{P}<0.05 \mathrm{com}-$ pared with $\mathrm{A},{ }^{\text {}} \mathrm{P}<0.01$; error bars represent the mean + standard deviation. IOD, integrated optical density.

secondary antibody (1:5,000; ZB-2305; ZSGB-BIO). The expression of GAPDH was detected as an internal control and the relative content of target proteins was analyzed using an enhanced chemiluminescence system (BeyoECL Plus; Beyotime Institute of Biotechnology, Nanjing, China).

RNA isolation and reverse transcription quantitative polymerase chain reaction ( $R T-q P C R)$ amplification of whole lung $m R N A$. To extract RNA from the lungs, $100 \mathrm{mg}$ frozen lung tissue was homogenized with $1 \mathrm{ml}$ TRIzol (Ambion, Life Technologies, Carlsbad, CA, USA) in a ribonuclease-free tube at $4^{\circ} \mathrm{C}$. RNA isolation was performed according to the manufacturer's instructions. Following extraction, total 

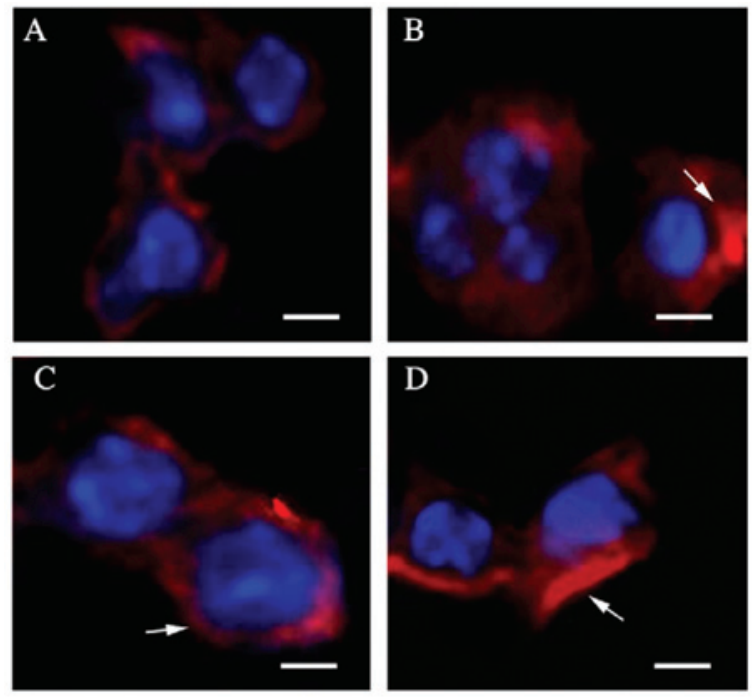

$\mathbf{E}$

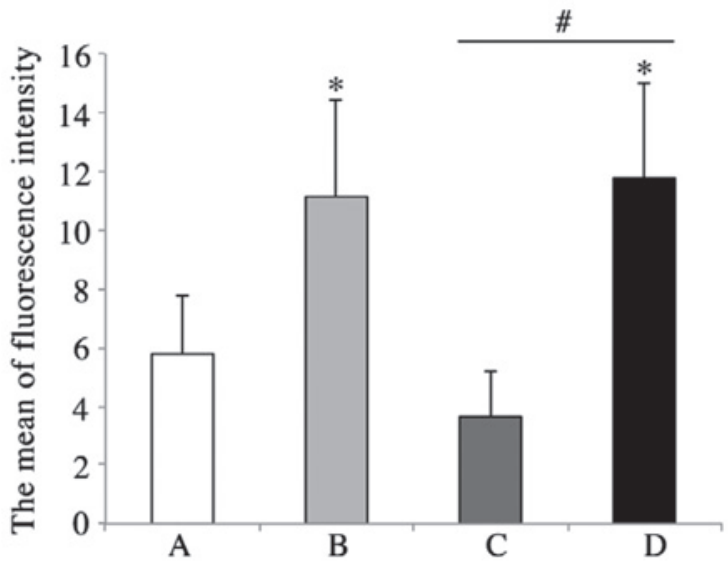

Figure 4. K. pneumoniae 03183 induces actin polymerization in lung tissue (magnification, $\mathrm{x} 400$; scale bar, $10 \mu \mathrm{m}$ ). (A) Phosphate-buffered saline-treated group; (B) group inoculated with bovine serum albumin-treated K. pneumoniae 03183; (C) group inoculated with high-mobility group nucleosome-binding domain 2-treated K. pneumoniae 03183; (D) group inoculated with untreated $K$. pneumoniae 03183 . F-actin is stained red (white arrows indicate actin stress fibers) and nuclei are stained blue with DAPI (E) Quantification of A-D. ${ }^{*} \mathrm{P}<0.05$, relative to values of $\mathrm{A},{ }^{,} \mathrm{P}<0.01$; error bars represent the mean fluorescence intensity of F-actin + standard deviation.

RNA was subjected to reverse transcription by using a First Strand cDNA Synthesis kit from Thermo Fisher Scientific, according to the manufacturer's instructions. The final product complementary DNA (cDNA) was stored at $-20^{\circ} \mathrm{C}$. cDNA was amplified on a Bio-Rad CFX-96 real-time system using SYBR Green qPCR Master mix from Thermo Fisher Scientific. The following specific primers were used: LCN2, 5'-CCAGTTCGCCATGGTATTTTTC-3' (sense) and 5'-CACACTCACCACCCATTCAGTT-3' (anti-sense); CXCL1, 5'-GCTGGGATTCACCTCAAGAA-3' (sense) and 5'-TCTCCGTTACTTGGGGACAC-3' (anti-sense), IL-6, 5'-GAGGATACCACTCCCAACAGACC-3' (sense) and 5'-AAGTGCATCATCGTTGTTCATACA-3' (anti-sense), $\beta$-actin, 5'-CGGTTCCGATGCCCTGAGGCTCTT-3' (sense) and 5'-CGTCACACTTCATGATGGAATTGA-3' (anti-sense); all were purchased from Sangon Biotech (Shanghai, China). Relative expression of GAPDH was normalized to the $\beta$-actin levels.

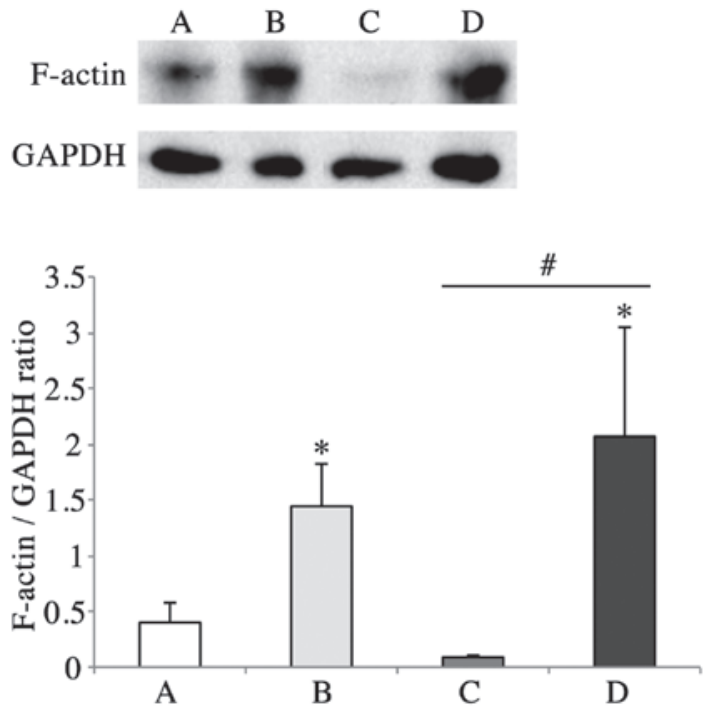

Figure 5. Western blot analysis of F-actin levels in lung tissue. (A) Phosphate-buffered saline-treated group; (B) group inoculated with bovine serum albumin-treated $K$. pneumoniae 03183; (C) group inoculated with high-mobility group nucleosome-binding domain 2-treated $K$. pneumoniae 03183; (D) group inoculated with untreated K. pneumoniae 03183. (E) Quantification of A-D. Error bars represent the mean + standard deviation of the F-actin levels normalized to GAPDH. ${ }^{*} \mathrm{P}<0.05$, relative to values of $\mathrm{A},{ }^{\#} \mathrm{P}<0.01$.

Statistical analysis. Values are expressed as the mean \pm standard deviation. Statistical analysis was performed using SPSS 13.0 (SPSS, Inc., Chicago, IL, USA). Statistical analyses were performed using the Student's t-test. $\mathrm{P}<0.05$ was considered to indicate a significant difference between values.

\section{Results}

HMGN2 reduces K. pneumoniae 03183 invasion. $128 \mu \mathrm{g} / \mathrm{ml}$ HMGN2 significantly inhibited the invasion of $K$. pneumoniae 03183 into lungs. 1\% BSA had no effect on the invasion of $K$. pneumoniae 03183 into lungs as compared with untreated K. pneumoniae 03183 (Fig. 1).

Pulmonary structure following K. pneumoniae 03183-inoculation. As illustrated in Fig. 2A, the pulmonary structure in the mice of the PBS group was normal. Although the alveoli of mice in the BSA group were remaining, the pulmonary structure exhibited dilatation of alveolar ducts, hyperemia and edema of alveolar walls, proliferation of uncharacteristic cells but no evidence of inflammatory cells (Fig. 2B). The condition of the pulmonary structure in the HMGN2 group was in a worse condition than that in the BSA group and exhibited excessive capillary leakage, accumulation of white blood cells in the alveolar spaces with diffuse damage of alveolar epithelial and endothelial cells (Fig. 2C). Lungs of mice in the $K$. pneumoniae group were similar to those in the BSA group; however, normal alveoli were not present (Fig. 2D).

Expression of F-actin in lung tissue. Positive staining for $\mathrm{F}$-actin expression was significantly increased in the mice in 
A

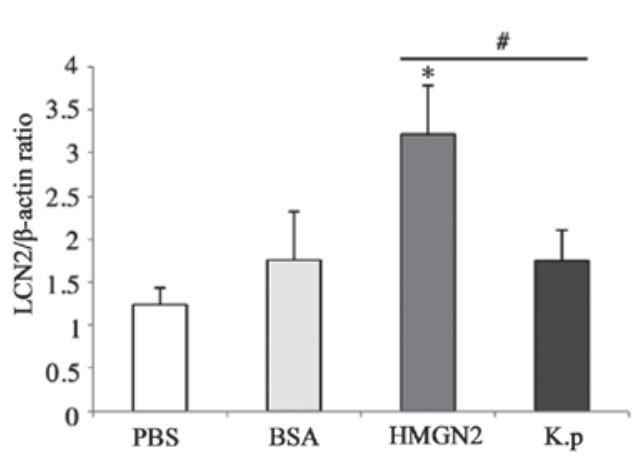

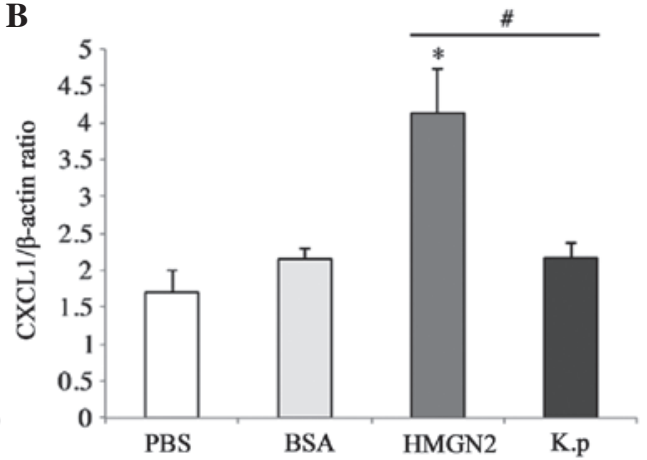

C

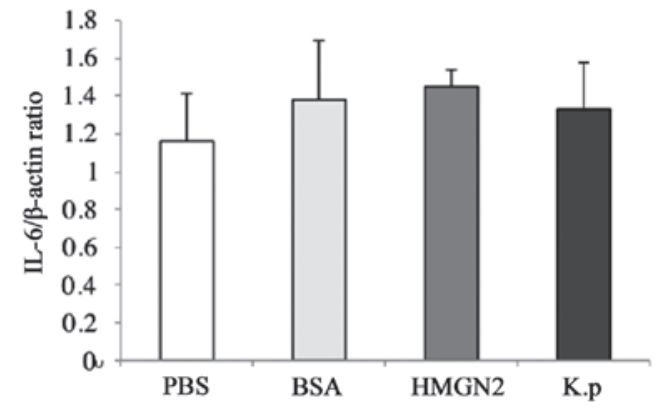

Figure 6. Reverse-transcription quantitative polymerase chain reaction analysis of (A) LCN2; (B) CXCL1 and (C) IL-6. ${ }^{*}<<0.05$, relative to PBS group, ${ }^{\#} \mathrm{P}<0.01$; error bars represent the mean + standard deviation. PBS group, group treated with phosphate-buffered saline; BSA, group inoculated with bovine serum albumin-treated K. pneumoniae 03183; HMGN2, group inoculated with high-mobility group nucleosome-binding domain 2-treated K. pneumoniae 03183; K.p, group inoculated with untreated K. pneumoniae 03183.

the BSA and $K$. pneumoniae groups, as compared with that in the mice in the HMGN2 group (Fig. 3).

HMGN2 reduces $K$. pneumoniae-induced actin fiber polymerization in pulmonary cells. As shown in Fig. 4A, in the pulmonary cells of mice in the PBS group, well-organized actin filaments were present. Actin stress fibers were observed at the cell periphery in most of the cells infected with $K$. pneumoniae 03183 pre-treated with $1 \%$ BSA (Fig. 4B). By contrast, pre-treatment with HMGN2 appeared to have eliminated the stress fibers and decreased the overall content of F-actin; most of the actin filaments were fragmented (Fig. 4C). Pulmonary cells of mice inoculated with untreated $K$. pneumoniae 03183 showed a similar F-actin fiber distribution to that in the BSA group (Fig. 4D). F-actin was quantified as the mean fluorescence intensity (Fig. 4E).

$K$. pneumoniae inoculation increases $F$-actin protein levels in lung tissue. To illustrate the changes in F-actin expression at the protein level, western blot analysis was employed to show the quantity of F-actin in the four different groups. Consistent with the results acquired by immunohistochemistry and immunofluorescence, F-actin levels in the BSA and $K$. pneumoniae groups were higher than those in the PBS group (Fig. 5). However, F-actin levels in the HMGN2 group were lower than those in the PBS group, which may indicate that HMGN2 decreased the quantity of F-actin generated by $\mathrm{G}$-actin polymerization.

HMGN2 affects K. pneumoniae 03183-induced inflammation-associated cytokines. To examine the effect of HMGN2 on $K$. pneumoniae 03183-induced inflammation-associated cytokine expression, RT-qPCR was performed to measure mRNA levels of the cytokines LCN2, CXCL1 and IL-6, which all have functions of restraining or killing $K$. pneumoniae 03183 (Fig. 6) $(12,13,15)$. Compared with levels in the PBS group, LCN2 and CXCL1 mRNA levels were higher in the BSA, HMGN2 and K. pneumoniae groups (Fig. 6A and $\mathrm{B}$ ). In the HMGN2 group, the mRNA levels of LCN2 and CXCL1 were more increased than those in the BSA and $K$. pneumoniae groups, which indicated that HMGN2 had an alleviating effect on $K$. pneumoniae infection. In the BSA, $K$. pneumoniae and HMGN2 groups, IL-6 levels were higher than those in the PBS group; however, the difference was not significant (Fig. 6C).

\section{Discussion}

The results of the present study indicated that HMGN2 was able to reduce $K$. pneumoniae 03183 invasion of host lung tissue by blocking $K$. pneumoniae 03183-induced actin polymerization and up-regulating the secretion of the inflammation-associated cytokines LCN2 and CXCL1.

Bacterial invasion to epithelial cells is a critical step in the manifestation of bacterial infection, in addition to evasion of the immune response of the organism and resistance to antibiotics (34). In the present study, bacteria adherent to epithelial cells were killed by the bactericidal antibiotic gentamicin, which is unable to enter eucaryotic cells, so that internalized organisms were protected from its effects (35). The results showed that HMGN2 significantly inhibited the invasion of K. pneumoniae 03183 into lung tissue in vivo compared with the invasion extent in the BSA and $K$.pneumoniae groups. The effect HMGN2 was observed at a concentration of $128 \mu \mathrm{g} / \mathrm{ml}$, which 
indicated that it inhibited the invasion of K. pneumoniae 03183 neither by inhibiting the growth of bacteria nor by inhibiting the physical progress of pre-treatment with protein $(24,25)$.

Altering the host cytoskeleton is crucial for mediating pathogen adherence, invasion and intracellular locomotion. Actin is the structure protein of the microfilament cytoskeleton, existing in two forms, monomer (G-actin) and multimer (F-actin). Normally, the progress of polymerization and depolymerization of microfilaments is balanced for the purpose of regulating actin activity, as only polymeric actin has biological effects $(7,8)$. In the present study, immunohistochemical techniques, fluorescence microscopy and western blot analysis were applied in order to observe the expression of F-actin in lungs and to determine the extent of depolymerization/polymerization of F-actin. The results of the present study showed that the recomposition of the pulmonary cytoskeleton was triggered by untreated $K$. pneumoniae 03183. In the $K$. pneumoniae and BSA groups, F-actin expression and stress fibers were increased and the average fluorescence intensity of F-actin was significantly increased as compared with that in the PBS group, which indicated that following infection, polymerized F-actin was enhanced. Of note, in the HMGN2 group, F-actin expression and average fluorescence intensity of F-actin were decreased, which indicated that HMGN2 inhibited K. pneumoniae 03183 invasion of lung cells directly through reducing the polymerization of F-actin, which is consistent with previous studies by our group $(24,25)$.

Cytokines and chemokines are critical molecules expressed in response to the presence of invading pathogens (10); they are involved in almost every facet of immunity and inflammation (11). Inflammation is the first response of the immune system to bacterial or viral infection (36). IL-6 has been found to enhance survival of pneumonia sepsis caused by K. pneumoniae via improving neutrophil killing (12). CXCL1, a member of the $\mathrm{C}-\mathrm{X}-\mathrm{C}$ chemokine family, is broadly produced by numerous cells, including fibroblasts, endothelial cells, as well as peritoneal and alveolar MФ (13). It was shown to be able to enhance resistance to $K$. pneumonia in mice $(13,14)$. LCN2, produced by epithelial cells and macrophages, has been identified as a potent bacteriostatic agent, inhibiting bacterial growth by sequestering the iron-binding bacterial siderophores and blocking bacterial access to iron $(15,16)$. In the present study, hematoxylin and eosin staining showed that the lung structure in the HMGN2 group was in a worse condition than that in the BSA and K. pneumoniae groups, which may indicate that HMGN2 generates a brisk innate immune response to K. pneumonia 03183 more rapidly. Furthermore, the results showed the levels of LCN2 and CXCL1 mRNA in the HMGN2 group were higher than those in the PBS, BSA, and $K$. pneumoniae groups, which may indicate that pre-treatment of $K$. pneumoniae neumonia with HMGN2 was able to improve the ability of the host to recognize $K$. pneumoniae and to secrete associated cytokines. However, no significant effect on IL-6 levels in the lung was noted.

In conclusion, HMGN2 inhibited the invasion of $K$. pneumoniae 03183 into lungs through reducing the polymerization of F-actin and increasing the levels of CXCL1 and LCN2 in vivo, which indicated that HMGN2 may be an important defense factor in the innate immune response.

\section{Acknowledgements}

China National Nature Science Fund 30671963 and China Medical Board of New York INC98-681.

\section{References}

1. Jarvis WR and Martone WJ: Predominant pathogens in hospital infections. J Antimicrob Chemother 29: 19-24, 1992.

2. Carpenter JL: Klebsiella pulmonary infections: occurrence at one medical center and review. Rev Infect Dis 12: 672-682, 1990.

3. Podschun R and Ullmann U: Klebsiella spp. as Nosocomial pathogens: Epidemiology, taxonomy, typing methods, and pathogenicity factors. Clin Microbiol Rev 11: 589-603, 1998.

4. Song JM, Bishop BL, Li GJ, Duncan MJ and Abraham SN: TLR4 initiated and CAMP mediated abrogation of bacterial invasion of the bladder. Cell Host Microbe 1: 287-298, 2007.

5. Gruenheid S and Finlay BB: Microbial pathogenesis and cytoskeletal function. Nature 422: 775-81, 2003.

6. Winder SJ and Ayscough KR: Actin-binding proteins. J Cell Sci 118: 651-654, 2005.

7. Tsai KW, Lai HT, Tsai TC, et al: Difference in the regulation of IL- 8 expression induced by uropathogenic $E$. coli between two kinds of urinary tract epithelial cells. J Biomed Sci 16: 91, 2009.

8. Bhavsar AP, Guttman JA and Finlay BB: Manipulation of host-cell pathways by bacterial pathogens. Nature 449: 827-834, 2007.

9. Haglund CM and Welch MD: Pathogens and polymers: microbe-host interactions illuminate the cytoskeleton. J Cell Biol 195: 7-17, 2011

10. Ye P, Garvey PB, Zhang P, et al: Interleukin-17 and lung host defense against Klebsiella pneumoniae infection. Am J Respir Cell Mol Biol 25: 335-340, 2001.

11. Borish LC and Steinke JW: Cytokines and chemokines. J Allergy Clin Immunol 111 (2 Suppl): S460-S475, 2003.

12. Sutherland RE, Olsen JS, McKinstry A, et al: Mast cell IL-6 improves survival from Klebsiella pneumonia and sepsis by enhancing neutrophil killing. J Immunol 181: 5598-5605, 2008.

13. Tsai WC, Strieter RM, Wilkowski JM, Bucknell KA, Burdick MD, Lira SA and Standiford TJ: Lung-specific transgenic expression of $\mathrm{KC}$ enhances resistance to Klebsiella pneumoniae in mice. J Immunol 161: 2435-2440, 1998.

14. Cai S, Batra S, Lira SA, Kolls JK and Jeyaseelan S: CXCL1 regulates pulmonary host defense to Klebsiella infection via CXCL2, CXCL5, NF-kappaB, and MAPKs. J Immunol 185: 6214-6225, 2010.

15. Goetz DH, Holmes MA, Borregaard N, et al: The neutrophil lipocalin NGAL is a bacteriostatic agent that interferes with siderophore-mediated iron acquisition. Mol Cell 10: 1033-1043, 2002.

16. Flo TH, Smith KD, Sato S, et al: Lipocalin 2 mediates an innate immune response to bacterial infection by sequestrating iron. Nature 432: 917-921, 2004.

17. Ganz T: Defensins: antimicrobial peptides of innate immunity. Nat Rev Immunol 3: 710-720, 2003.

18. Shirakawa H, Herrera JE, Bustin M and Postnikov Y: Targeting of high mobility group-14/-17 proteins in chromatin is independent of DNA sequence. J Biol Chem 275: 37937-37944, 2000.

19. Subramanian M, Gonzalez RW, Patil H, et al: The nucleosome-binding protein HMGN2 modulates global genome repair. FEBS J 276: 6646-6657, 2009.

20. Feng Y, Huang N, Wu Q and Wang B: HMGN2: a novel antimicrobial effector molecule of human mononuclear leukocytes? J Leukocyte Biol 78: 1136-1141, 2005.

21. Feng Y, Huang N, Wu Q, Bao L and Wang BY: $\alpha$-helical domain is essential for antimicrobial activity of high mobility group nucleosomal binding domain 2 (HMGN2). Acta Pharmacol Sin 26: 1087-1092, 2005.

22. Feng Y, He F, Zhang P, et al: Inhibitory effect of HMGN2 protein on human hepatitis $\mathrm{B}$ virus expression and replication in the HepG2.2.15 cell line. Antiviral Res 81: 277-282, 2009.

23. Xiong WB, Hung N, Feng Y, Wu Q and Wang BY: Creation and anti-cancer potency in HeLa cells of a novel chimeric toxin, HMGNCIDIN, composed of HMGN2 $\alpha$-helical domain and PE38 KDEL domain III. Chin Med J (Engl) 121: 82-85, 2008. 
24. Wu G, Cao Y, Fan B, et al: High-mobility group protein N2 (HMGN2) inhibited the internalization of Klebsiella pneumoniae into cultured bladder epithelial cells. Acta Biochim Biophys Sin (Shanghai) 43: 680-687, 2011.

25. Cao Y, Wu GX, Fan B, et al: High mobility group nucleosomal binding domain 2 protein protects bladder epithelial cells from Klebsiella pneumoniae invasion. Biol Pharm Bull 34: 1065-1071, 2011.

26. Andersson U, Wang $\mathrm{H}$, Palmblad K, et al: High mobility group 1 protein (HMG-1) stimulates proinflammatory cytokine synthesis in human monocytes. J Exp Med 192: 565-570, 2000.

27. Ulloa L, Batliwalla FM, Andersson U, et al: High mobility group box chromosomal protein 1 as a nuclear protein, cytokine, and potential therapeutic target in arthritis. Arthritis Rheum 48: 876-881, 2003.

28. Pisetsky DS, Erlandsson-Harris H and Andersson U: High-mobility group box protein 1 (HMGB1): an alarmin mediating the pathogenesis of rheumatic disease. Arthritis Res Ther 10: 209, 2008.

29. Yang D, Postnikov YV, Li Y, et al: High-mobility group nucleosome-binding protein 1 acts as an alarmin and is critical for lipopolysaccharide- induced immune responses. J Exp Med 209: 157-171, 2012.
30. Mills B, Arrington JB and Sobin LH: Laboratory methods in histotechnology. Washington, DC: American registry of pathology 132-214, 1992.

31. Deng LX, Wu GX, Cao Y, et al: The chromosomal protein HMGN2 mediates the LPS-induced expression of $\beta$-defensins in mice. Inflammation 35: 456-473, 2012.

32. Li QQ, Chen ZQ, Cao XX, et al: Involvement of $\mathrm{NF}-\kappa \mathrm{B} / \mathrm{miR}-448$ regulatory feedback loop in chemotherapy-induced epithelial-mesenchymal transition of breast cancer cells. Cell Death Differ 18: 16-25, 2011.

33. Nohara A, Okada S, Ohshima K, et al: Cyclin-dependent kinase- 5 is a key molecule in tumor necrosis factor- $\alpha$-induced insulin resistance. J Biol Chem 286: 33457-33465, 2011

34. Lafont F and van der Goot FG: Bacterial invasion via lipid rafts. Cell Microbiol 7: 613-620, 2005.

35. Kihlström E and Andåker L: Inability of gentamicin and fosfomycin to eliminate intracellular Enterobacteriaceae. J Antimicrob Chemother 15: 723-728, 1985.

36. Snelgrove R, Gwyer E and Hussell T. Modulation of immunity to respiratory viral infection. Future Virol 1: 471-481, 2006. 